\title{
MEG-EEG Fusion by Kalman Filtering within a Source Analysis Framework*
}

\author{
Laith Hamid $^{1}$, Ümit Aydin ${ }^{2}$, Carsten Wolters ${ }^{2}$, Ulrich Stephani ${ }^{1}$, Michael Siniatchkin ${ }^{3}$ and Andreas Galka ${ }^{1}$.
}

\begin{abstract}
The fusion of data from several neuroimaging modalities may improve the temporal and spatial resolution of non-invasive brain imaging. In this paper, we present a novel method for combining simultaneous electroencephalographic (EEG) and magnetoencephalographic (MEG) measurements within the framework of source analysis. This method represents an extension of a previously published spatio-temporal inverse solution to the case of MEG or combined MEGEEG signals. Moreover, we use a state-of-the-art realistic finite element (FE) head model especially calibrated for the MEGEEG fusion problem. Using a real data set containing an epileptic spike, we compare the source analysis results of the spatio-temporal inverse solution to the results of the LORETA method and to the findings from other structural and functional modalities. We show that the proposed fusion method, despite the low signal-to-noise ratio (SNR) of single spikes, produces comparable results to the other modalities. Furthermore, it correctly identifies the same source as the main generator for the MEG and EEG spikes.
\end{abstract}

\section{INTRODUCTION}

Simultaneous recording of the electroencephalogram (EEG) and the magnetoencephalogram (MEG) offers a good example of multimodal imaging of the human brain. If used independently, each modality produces measurements with high temporal resolution and relatively low spatial resolution. In order to improve the spatial resolution, source analysis may be used to reconstruct the brain activity from surface measurements. Since EEG and MEG signals offer complementary information about the same neuronal generators in the brain, an even better spatial resolution is achieved by combining the signals from both modalities within a source analysis framework. Multimodal imaging by MEG-EEG fusion results in a more stable reconstruction of brain sources and opens the way to a wider application of these imaging techniques in brain research and clinical neuroscience and especially in the pre-surgical evaluation of epilepsy patients.

Source analysis aims at imaging the brain using surface EEG and MEG measurements and requires the solution of the MEG-EEG forward and inverse problems. The MEG-EEG

\footnotetext{
*This work was supported by the German Research Foundation (DFG) through the Collaborative Research Center (SFB) 855 "Biomagnetic Sensing" and DFG project WO1425/2-1

${ }^{1}$ Laith Hamid, Ulrich Stephani and Andreas Galka are with the Department of Neuropediatrics, University of Kiel, 24098 Kiel, Germany. \{lah, stephani, a.galka\} at pedneuro.uni-kiel.de

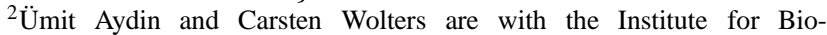
magnetism and Biosignal Analysis, University of Münster, D-48149 Münster, Germany. \{umit.aydin, carsten.wolters\} at uni-muenster.de

${ }^{3}$ Michael Siniatchkin is with the Department of Psychiatry, Psychosomatic Medicine and Psychotherapy, University of Frankfurt, 60528 Frankfurt am Main, Germany. michael.siniatchkin at kgu.de
}

forward problem consists of the calculation of the electric potentials, in case of EEG, or the magnetic fields, in case of MEG, on the head surface resulting from the activity of a set of current dipoles in the brain with known location, orientation and strength. The solution requires a model for the geometries and conductivities of the intervening head tissues. It has been shown that EEG is affected more by the conductivity and geometry of the skin and skull in comparison to MEG, while both of them are affected by the properties of tissues inside skull (including inner skull surface and anisotropy) [1]. Thus in order to combine those two modalities the sensitivity differences should be considered using a sufficiently elaborate forward model. To this end we use a 6-compartment anisotropic finite element (FE) head model in this study.

The MEG-EEG inverse problem consists of estimating the current density in the brain which generated a given set of EEG or MEG recordings, or both, for a given head model. Since the number of measurements is much smaller than the number of sources, the inverse problem becomes illposed and results in an infinite number of solutions. One possibility to stabilize a solution is achieved by the LORETA method [2], which imposes a spatial smoothness constraint in order to regularize the inverse problem. This kind of constraint, however, still ignores the time series aspect of the measurements and the information contained therein. As a remedy to this problem, a spatio-temporal solution to the EEG inverse problem was presented in [3], based on an estimation technique known as spatio-temporal Kalman filtering (ST-KF). In this study, we propose an extension of the aforementioned method to the case of simultaneous MEG-EEG measurements.

\section{Methods}

The measurement process can be modeled with the following equation:

$$
\mathbf{Y}_{k}=\mathrm{K}_{s} \mathbf{J}_{k}+\varepsilon_{k}
$$

where $\mathbf{Y}=\left[\begin{array}{ll}\mathbf{Y}_{M} & \mathbf{Y}_{E}\end{array}\right]^{T}$ describes the $\left(N_{Y} \times 1\right)$-dimensional measurement vector, where $N_{Y}$ is the total number of the $N_{M}$ MEG and the $N_{E}$ EEG sensors. $\mathbf{J}$ denotes the current density vector with $N_{J}$ sources, defined on the 3D source grid discretized from the brain's gray matter. Each of the $N_{v}$ points of the source grid corresponds to the location of the current density vector $\mathbf{j}_{v}=\left[\begin{array}{lll}j_{x} & j_{y} & j_{z}\end{array}\right]^{T}$ which leads to $N_{J}=3 N_{v}$ sources. $\mathrm{K}_{s}$ is the $\left(N_{Y} \times N_{J}\right)$-dimensional lead field matrix (LFM) which results from the solution ot the MEG- 
EEG forward problem. This matrix is defined as

$$
\mathrm{K}_{s}=\left[\begin{array}{c}
\mathrm{S}_{M} \mathrm{~K}_{M} \\
\mathrm{~S}_{E} \mathrm{~K}_{E}
\end{array}\right]
$$

where $S_{M}=s_{Y M} I_{N_{M}}$ and $S_{E}=s_{Y E} I_{N_{E}}$ denote the LFM-scaling matrices for MEG and EEG respectively; $s_{Y M}$ and $s_{Y E}$ are the MEG and EEG scaling factors which will be estimated from the measurements. $\mathrm{K}_{M}$ is the $\left(N_{M} \times N_{J}\right)$-dimensional MEG-LFM while $\mathrm{K}_{E}$ is the $\left(N_{E} \times N_{J}\right)$-dimensional EEGLFM. $\varepsilon=\left[\begin{array}{ll}\varepsilon_{M} & \varepsilon_{E}\end{array}\right]^{T}$ describes the $\left(N_{Y} \times 1\right)$-dimensional white Gaussian measurement noise. Furthermore, it is assumed that the measurement noise has zero mean and a diagonal $\left(N_{Y} \times\right.$ $N_{Y}$ )-dimensional covariance matrix

$$
\Sigma_{\varepsilon}=\left[\begin{array}{cc}
\Sigma_{\varepsilon M} & \underline{0} \\
\underline{0} & \Sigma_{\varepsilon E}
\end{array}\right]
$$

where $\Sigma_{\varepsilon M}=\sigma_{\varepsilon M}^{2} \mathrm{I}_{N_{M}}$ and $\Sigma_{\varepsilon E}=\sigma_{\varepsilon E}^{2} \mathrm{I}_{N_{E}}$. The rest is padded with zero matrices. For a more detailed description we refer to [3] and [4].

\section{A. LORETA}

The LORETA method employs a spatial smoothness constraint to penalize spatially non-smooth source configurations. At each time instant $k$, the estimated current density is computed using:

$$
\hat{\mathbf{J}}_{k}=\min _{\mathbf{J}}\left(\left\|\mathbf{Y}_{k}-\mathrm{K}_{s} \mathbf{J}_{k}\right\|^{2}+\lambda^{2}\left\|\mathrm{~L} \mathbf{J}_{k}\right\|^{2}\right)
$$

where $\lambda^{2}$ is the regularization parameter, in this paper obtained by minimizing the the Akaike Bayesian Information Criterion (ABIC)[3] ; L describes the $\left(N_{J} \times N_{J}\right)$-dimensional discrete spatial Laplacian operator, which can be thought of as a discrete approximation to the second-order spatial derivative.

\section{B. Spatio-temporal Kalman Filter}

In order to impose a temporal smoothness constraint, in addition to the spatial smoothness constraint of LORETA, a model for the evolution of the current density in space and time is described by the system equation:

$$
\mathbf{J}_{k}=\mathrm{A} \mathbf{J}_{k-1}+\eta_{k}
$$

where $\mathrm{A}$ is the $\left(N_{J} \times N_{J}\right)$-dimensional state transition matrix and $\eta$ represents the $\left(N_{J} \times 1\right)$-dimensional system noise. Let $\Sigma_{\eta}$ denote the $\left(N_{J} \times N_{J}\right)$-dimensional system noise covariance matrix. (1) and (2) represent a linear state space model, such that the inverse problem is recast into a state estimation problem. The reconstruction of the state $\mathbf{J}$ is then performed using a modified Kalman filter.

Due to the high dimension of the state vector, additional assumptions need to be made to simplify the computation of the state estimates. First, we multiply the system equation with the discrete Laplacian operator $L$ to effect a spatial decoupling of the states and to obtain an approximately diagonal system noise covariance matrix:

$$
\tilde{\mathbf{J}}=\mathrm{L} \mathbf{J}
$$

In the following, we will drop the tilde from our notation but it should be understood that all equations refer to a laplacianized state space. Additionally, we assign the same system noise variance to all grid points:

$$
\Sigma_{\eta}=\sigma_{\eta}^{2} \mathrm{I}_{N_{j}}
$$

By the spatial whitening operation of (3) a practically intractable high-dimensional state estimation problem is transformed into a set of low-dimensional local state estimation problems, located at each grid point $v$. The interaction between grid points is limited to immediate neighbors. We simplify the model further by assuming the state transition matrix to obey

$$
\mathrm{A}=a_{1} \mathrm{I}_{N_{J}}-b_{1} \mathrm{~L}
$$

thus assigning the same self-dynamics parameter $a_{1}$ and the same neighbor-dynamics parameter $b_{1}$ to all grid points, and at each grid point to all three current components. Within (2) the time lag both for self- and neighbor dynamics is limited to 1 , and only immediate neighbors are coupled.

Each temporal iteration step of the ST-KF begins by estimating, for each grid point $v$, the predicted state estimate:

$$
\hat{\mathbf{j}}_{v, k \mid k-1}=\left(a_{1} I_{3}\right) \hat{\mathbf{j}}_{v, k-1 \mid k-1}+\frac{1}{6}\left(b_{1} I_{3}\right) \sum_{v^{\prime} \in \mathscr{N}(v)} \hat{\mathbf{j}}_{v^{\prime}, k-1 \mid k-1}
$$

where $\mathscr{N}(v)$ denotes the set of grid points which are immediate neighbors of $v$. The initial state estimate $\hat{\mathbf{j}}_{v, 1 \mid 1}$ is chosen to be zero. The respective state prediction covariance matrix is given by

$$
\mathrm{p}_{v, k \mid k-1}=\left(a_{1} \mathrm{I}_{3}\right) \mathrm{p}_{v, k-1 \mid k-1}\left(a_{1} \mathrm{I}_{3}\right)^{T}+\sigma_{\eta}^{2} \mathrm{I}_{3}
$$

Globally, the predicted measurement follows as

$$
\hat{\mathbf{Y}}_{k}=\overline{\mathrm{K}}_{s} \hat{\mathbf{J}}_{k \mid k-1}
$$

where $\overline{\mathrm{K}}_{s}$ denotes the laplacianized LFM $\mathrm{K}_{s} \mathrm{~L}^{-1}$. Now the difference between the actual measurement and the predicted measurement represents the measurement prediction error or innovation

$$
\mathbf{R}_{k}=\mathbf{Y}_{k}-\hat{\mathbf{Y}}_{k}
$$

The innovation covariance matrix is then given by

$$
\Sigma_{R}=\sum_{v=1}^{N_{v}} \overline{\mathrm{k}}_{s}(v) \mathrm{p}_{v, k \mid k-1} \overline{\mathrm{k}}_{s}(v)^{T}+\Sigma_{\varepsilon}
$$

Here $\overline{\mathrm{k}}_{s}(v)$ represents the contribution of the source at grid point $v$ to the measurements. Consequently, the local Kalman gain is given by

$$
\mathrm{g}_{v, k}=\mathrm{p}_{k \mid k-1} \overline{\mathrm{k}}_{s}(v) \Sigma_{R}^{-1}
$$

$\mathrm{g}_{v, k}$ can be interpreted as a local inverse of the LFM. Finally, the corrected state estimate at grid point $v$ is given by

$$
\hat{\mathbf{j}}_{v, k \mid k}=\hat{\mathbf{j}}_{v, k \mid k-1}+\mathrm{g}_{v, k} \mathbf{R}_{k}
$$

and its corresponding local state estimation covariance matrix is given by

$$
\mathrm{p}_{v, k \mid k}=\left(\mathrm{I}_{3}-\mathrm{g}_{v, k} \overline{\mathrm{k}}_{s}(v)\right) \mathrm{p}_{v, k \mid k-1}
$$


With (6) and (7) one loop of the temporal iteration step of the ST-KF is completed, and the next loop starts again from (4) and (5), replacing $k$ by $k+1$.

After all time points have been processed by ST-KF, the global state estimates need to be transformed back to the delaplacianized state space, using $\mathrm{L}^{-1} \mathbf{J}$ and $\mathrm{L}^{-1} \mathrm{P}\left(\mathrm{L}^{T}\right)^{-1}$.

\section{Parameter Estimation}

The optimal parameters, in the maximum likelihood sense, are estimated by minimizing the Akaike information criterion (AIC) as given by

$$
\begin{array}{r}
A I C\left(a_{1}, b_{1}, s_{Y M}, S_{Y E}, \sigma_{\varepsilon M}^{2}, \sigma_{\varepsilon E}^{2}\right)=-2 \sum_{k=1}^{N_{k}}\left(\mathbf{R}_{k} \Sigma_{R}^{-1} \mathbf{R}_{k}\right. \\
\left.+\log \left|\Sigma_{R}\right|+N_{Y} \log (2 \pi)\right)+2 N_{\text {par }}
\end{array}
$$

where $N_{k}$ is the number of time points in the data and $N_{\text {par }}$ is the number of parameters, in this case 6. The LFM scaling factors $s_{Y M}, s_{Y E}$ introduced above and the system noise covariance parameter $\sigma_{\eta}^{2}$ should not be optimized simultaneously since they represent the same degree of freedom. We find it more convenient to assign this freedom to the LFM and to keep $\sigma_{\eta}^{2}$ fixed at 1 . We remark that the introduction of separate parameters for MEG and EEG, instead of just single parameters for LFM scaling and measurement noise covariance, represents a major modification of the original ST-KF algorithm. It is through this modification that MEGEEG-fusion becomes possible.

The optimization problem is solved by iteratively by alternating between the Broyden-Fletcher-Goldfarb-Shanno (BFGS) secant and the Nelder-Mead simplex methods, in order to avoid local minima. Since application of each method requires multiple runs of the Kalman filter, the parameter optimization step is, with respect to the computational time, the most expensive part of the method.

\section{Head Model}

The head model was constructed using T1w $(1.17 \times$ $\left.1.04^{2} \mathrm{~mm}^{3}\right), \quad \mathrm{T} 2 \mathrm{w}\left((1.17 \mathrm{~mm})^{3}\right)$ and diffusion weighted (DW) $\left((1.875 \mathrm{~mm})^{3}\right)$ magnetic resonance images (MRI) acquired with a Gyroscan Intera/Achieva system (3.0 Tesla, System Release 2.5; Philips, Best, NL). The DW-MRI was measured according to Stejskal-Tanner spin-echo EPI sequence with a SENSE parallel imaging scheme in AP direction (acceleration factor 2). 20 volumes with diffusion sensitivity $b=1000 \mathrm{~S} / \mathrm{mm}^{2}$ using diffusion weighted gradients in 20 directions, equally distributed on a sphere, were acquired along with a $b=0 \mathrm{~S} / \mathrm{mm}^{2}$ volume. Another $b=0 \mathrm{~S} / \mathrm{mm}^{2}$ volume with reversed encoding gradients was measured to be used in susceptibility correction.

T1w and T2w images were rigidly registered to each other and used to segment the inner skull, outer skull and skin using the FSL-BETSURF function [6]. White matter (WM), gray matter (GM) and cerebro-spinal fluid (CSF) were segmented using the FSL-FAST algorithm. To distinguish between skull spongiosa and compacta, the skull estimate was eroded by one pixel and a threshold-based segmentation algorithm was used on the $\mathrm{T} 2 \mathrm{w}$ image constrained by the eroded skull.

The DW-MRIs were used to obtain WM and GM anisotropy. After eddy current correction using FSL, the images were corrected for susceptibility artifacts using the two $b=0$ images with reversed encoding gradients, using the FAIR toolbox [5]. Consequently, the DW-MRIs were registered to the $\mathrm{T} 2 \mathrm{w}$ image and the FSL-DTIFIT function was used to construct diffusion tensors. The conductivity tensors were estimated from the diffusion tensors as explained in [1]. Then a 6-compartment geometrically adapted hexahedral mesh with anisotropic WM and GM was built with SimBioVGRID. The LFMs for EEG and MEG were calculated using the SimBio software with conductivitity values $0.43,0.007$, $0.025,0.14,0.33$ and $1.79 \mathrm{~S} / \mathrm{mm}$ for skin, skull compacta, skull spongiosa, WM, GM and CSF, respectively [7].

\section{Application ExAmple: EPILEPTiC SPIKe ANALYSIS}

The ST-KF method was applied to MEG-EEG data from a 19 years old female with severe drug-resistant epilepsy caused by focal cortical dysplasia in the left mesial temporal region. Epilepsy started at the age of 6 with auras, complex focal seizures and secondary generalized tonic clonic seizures. The patient had received 15 different anti-epileptic drugs but never reached seizure freedom. The inter-ictal EEG recordings showed continuous epileptiform discharges over the left fronto-temporal region (electrodes FT9, F9 and T9). Ictal EEG recording showed ictal activity in the left frontal region. Positron emission tomography (PET) showed hypometabolism in the left mesial temporal region. We used the findings of structural and functional neuroimaging, as well as results from LORETA, to validate the source analysis results of the ST-KF.

The patient underwent 275-channel whole head MEG (CTF, VSM MedTech Ltd.) and simultaneous 74-channel EEG recording in a magnetically shielded room. The data set included 500 seconds sampled at $1.2 \mathrm{KHz}$. Experienced neurophysiologists inspected the MEG-EEG data and marked the epileptic spikes common to EEG and MEG. The number of channels was then reduced to 64 EEG and $64 \mathrm{MEG}$ channels, in order to save computational time. A 4-seconds segment containing, after a 2 -seconds pre-spike period, a single FT9 spike, was selected for source analysis with LORETA and ST-KF. The shape of the EEG spike appeared more smeared than the shape of the corresponding MEG spike; the MEG spike reached its peak $20 \mathrm{~ms}$ earlier than the EEG spike. This may be explained by the attenuation and smearing effects of volume conduction on the EEG signal and by the different sensitivities of EEG and MEG to tangential and radial source components. The MEG and EEG channels with the strongest spike amplitude are depicted in Fig. 1.

For comparison purposes, we created three data sets including only the 64 EEG channels, only the 64 MEG channels and the combined 128 MEG-EEG channels. For the analysis by ST-KF, we used, for each data set, the 2-second 


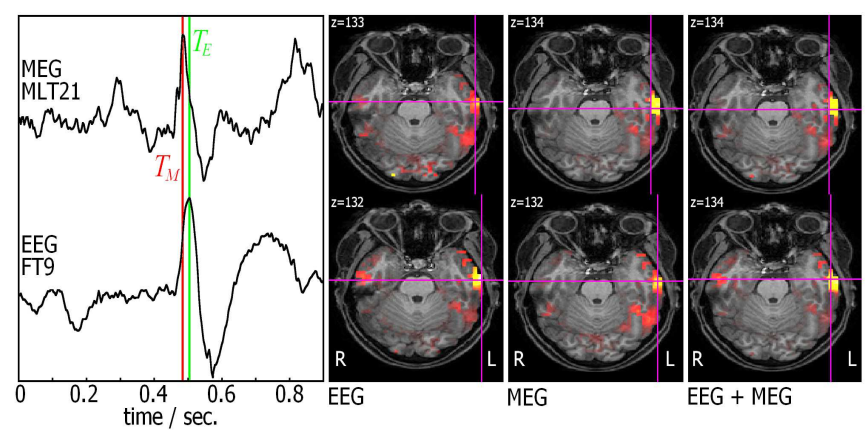

Fig. 1. The left figure shows the MEG and EEG channels with the strongest spikes, where the red and green lines mark the MEG and EEG spike maxima, respectively. The right figure summarizes the ST-KF source analysis results for the EEG, MEG and combined MEG-EEG data sets. The upper row displays the localization results at time $T_{M}$, while the lower row shows results at time $T_{E}$. The axial slices are displayed with their respective $\mathrm{z}$ coordinates in MRI space, which increases in the upward direction; the strongest activation is color-coded in yellow.

pre-spike period for parameter estimation and performed optimization until convergence of AIC. Furthermore, we computed the current density estimates using LORETA. In order to compare the source analysis results, we used the spike maxima from the MEG at time $T_{M}$ and the EEG at time $T_{E}$, respectively (see Fig. 1). Visualizations were created with the fieldtrip toolbox[8].

Using the MEG data set, at $T_{M}$ both methods localized the source in the left lateral temporal lobe. Likewise for the EEG data set, at $T_{E}$ both methods found a source in a very similar location. Using the combined MEG-EEG data set, LORETA and ST-KF correctly assigned the sources at the respective spike maxima to the same regions as in the MEG and EEG data sets. Furthermore, further activations in other regions of the brain were considerably weakened. Fig. 1 shows the localization results at times $T_{M}$ and $T_{E}$ for the EEG, MEG and combined MEG-EEG data sets using the ST-KF.

For ST-KF, AIC values were, for all three data sets, substantially smaller than the corresponding ABIC values of LORETA. From this it follows that the ST-KF, in the statistical sense, explains the data better than LORETA. Additionally, the source waveforms resulting from the STKF method displayed higher temporal smoothness than the respective waveforms resulting from LORETA. The explanation for these results comes from the additional temporal smoothness constraint used by the ST-KF in order to regularize the inverse solution.

The single example provided above demonstrated the practicality of MEG-EEG fusion using ST-KF source analysis and the plausibility of the localization, compared to the results of other neuroimaging modalities. However, more spikes need to be analyzed in order to obtain better estimates of the sources generating the epilepsy in this case.

\section{CONCLUSION}

In this paper we have addressed the problem of fusion of simultaneously recorded MEG and EEG. We have demonstrated that state space modeling provides a natural frame- work for such fusion tasks, since it links the two different recordings to a common source space, representing the neural generators. Through the corresponding parameter matrices of the state space model, both the spatial and the temporal domain are represented well within the model. We have shown that detailed individual anatomical knowledge can be incorporated conveniently into state space modeling by employing the corresponding LFMs as observation matrices of the model. LFMs were modeled by a state-of-the-art 6compartment anisotropic FE model. Those parameters of the state space model for which no prior information is available, can be estimated from the data by minimum-AIC, a well established statistical criterion.

The main difference to previous methods for estimating neural sources, like LORETA, is given by the fact that state space modeling makes explicit use of the temporal ordering of the data, while LORETA results would be invariant under temporal permutation. This enables us to use the available information to much better extent.

We have demonstrated the practicality of the proposed method by analyzing a single inter-ictal epileptic spike. The ST-KF successfully assigned the spikes in MEG and EEG to the same generating source within the brain, and the results were comparable to those of other structural and functional imaging modalities, like PET and MRI.

In the future we will analyze more inter-ictal spikes from several patients. We will also investigate the effects of including more MEG and EEG channels into the analysis. Furthermore we intend to improve the temporal regularization by using more detailed dynamical models, employing prior knowledge from brain physiology, and by adapting the system parameters in space and time. The validation of clinical data will also be improved by using post-surgical results and invasively measured electrophysiological data.

\section{REFERENCES}

[1] M. Rullmann, A. Anwander, M. Dannhauer, S.K. Warfield, F.H. Duffy, C.H. Wolters, EEG source analysis of epileptiform activity using a $1 \mathrm{~mm}$ anisotropic hexahedra finite element head model, Neuroimage vol. 44, pp. 399-410, 2001

[2] R.D. Pascual-Marqui, C.M. Michel, D. Lehmann. Low resolution electromagnetic tomography: a new method for localizing electrical activity in the brain, International Journal of Psychophysiology vol. 18, pp. 49-65, 1994

[3] A. Galka, O. Yamashita, T. Ozaki, R. Biscay, P. Valdes-Sosa, A solution to the dynamical inverse problem of EEG generation using spatiotemporal Kalman filtering, NeuroImage, vol. 23, Issue 2, pp. 435-453, 2004

[4] O. Yamashita, A. Galka, T. Ozaki, R. Biscay, P. Valdes-Sosa , Recursive penalized least squares solution for dynamical inverse problems of EEG generation, Human Brain Mapping, vol. 21, pp. 221-235, 2004

[5] L. Ruthotto, H. Kugel, J. Olesch, B. Fischer, J. Modersitzki, M. Burger, C.H. Wolters, Diffeomorphic Susceptibility Artefact Correction of Diffusion-Weighted Magnetic Resonance Images, Physics in Medicine and Biology, vol. 57, pp. 5715-5731, 2012

[6] M. Jenkinson, C.F. Beckmann, T.E.J. Behrens, M.W. Woolrich, S.M. Smith, FSL, NeuroImage, vol. 62-2, pp. 782-790, 2012

[7] SimBio: A generic environment for bio-numerical simulations https://www.mrt.uni-jena.de/simbio.

[8] R. Oostenveld, P. Fries, E. Maris, J.M. Schoffelen, FieldTrip: Open Source Software for Advanced Analysis of MEG, EEG, and Invasive Electrophysiological Data, Computational Intelligence and Neuroscience, vol. 2011, Article ID 156869, 9 pages, 2011 